\title{
Cone Beam Computed Tomography Used in the Assessment of the Alveolar Bone in Periodontitis
}

\author{
Alexandra Mihaela Stoica, Assistant Lecturer, DMD \\ Monica Monea, Associate Prof., DMD, PhD \\ Ramona Vlad, Postgraduate Student \\ Dragos Dan Sita, Assistant Lecturer, DMD
}

Department of Odontology and Oral Pathology, Faculty of Dental Medicine,

University of Medicine and Pharmacy Tirgu Mures, Romania

Mircea Buruian, Prof., $M D, P h D$

Department of Radiology, Faculty of Medicine,

University of Medicine and Pharmacy Tirgu Mures, Romania

doi: 10.19044/esj.2016.v12n27p47 URL:http://dx.doi.org/10.19044/esj.2016.v12n27p47

\begin{abstract}
Objectives: The aim of our study was to highlight the advantages of using Cone Beam Computed Tomography in the study of the extent of the alveolar bone loss, compared to the conventional intraoral radiography and to prove the boon of the CBCT scans for establishing the correct periodontal diagnosis.

Material and methods: A total of 16 patients with age between 35-55 years old, and a minimum of 8 teeth per dental arcade, presenting peridontal clinical symptomatology were selected. We used a custom periodontal chart that included the measuring of the gingival recession and the pocket depth in 6 points for 16 teeth, 8 maxillary teeth and 8 mandibulary teeth in all cases. For the radiographic evaluation we used CBCT imaging and intraoral radiography.

Results: CBCT scans offers the possibilities of measuring with accuracy the alveolar bone loss on mesial, distal vestibular and oral sides. It provides images with the exact position of the bone and also the expediency to assess the correct diagnosis. Retroalveolar radiography offers just a hint of the possible position of the alveaolar bone in all cases the anatomical details were offered by CBCT.

Conclusions: A correct periodontal diagnosis using conventional radiography is not possible because of the superimposition of the anatomical structures. The importance of CBCT imaging is no longer disputed, at the present time it is the best radiographic investigation available.
\end{abstract}


Keywords: Cone Beam Computed Tomography, alveolar bone, periodontal disease, Radiology

\section{Introduction}

Periodontal disease or Periodontitis is a chronic bacterial infection that affects the supporting anatomical structures of teeth. The decisive pathological sign is the resorbtion of the alveolar bone, followed by alteration of the gingival tissue. An accurate periodontal diagnostic and a flawless surgical treatment entails a radiological alveolar bone evaluation (Patel S. 2009). These common investigations are the intraoral retroalveolar or bite-wing radiography and Orthopantomogram. The paucity of information obtained is due to superimposition of the anatomical structures, these radiological investigation being two-dimensional scans (Durack $\mathrm{C}$ et al 2012). Intraoral and extraoral conventional radiographs, used distinct or combined suffer from the same inevitable limitations of all planar twodimension scans: magnification, distorsion and misrepresentation of all structures (Tyndall DA et al 2008). Computed Tomography (CT) has been available for a few years but the aplication in dentistry has been limited because of the cost, access and level of radiation dose which must be first taken in cosiderations (Agrawal P et al 2012).

The introduction of Cone Beam Computed Tomography (CBCT) was initially dedicated to angiography, but due to the information gain is used in maxillofacial imaging to perform the transition from two-dimentional to three-dimentional data reconstruction. In recent years (2013-2015) CBCT scans have been frequently used in dental field due to the detailed threedimensional images of the region of interest (Shekhar V et al 2013, Acar B et al 2014). A lower cost and radiation dose compared to the conventional Computed Tomography and the resource of obtaining images for a small anatomical region including two or three teeth, supports the use of CBCT. Offering a volumetric image increases the understanding of the location, severity and the source of the problem, especially in bone defects associated with periodontal disease or even endodontic lesions (Eshraghi T et al 2012, deFaria Vasconcelos K ey al 2012).

\section{I.}

\section{Material and methods}

The CBCT scans and the conventional X-rays were conducted for a total of 16 patients with age between 35-55 years old, and a minimum of 8 teeth per dental arcade, presenting peridontal clinical symptomatology. The isolated teeth were excluded from the study. The basic periodontal clinical symptomatology that all cases presented were: gingival recesion, tooth mobility, gingival swelling and bleeding. We used a custom periodontal 
chart that included the measurment of the gingival recession and the pocket depth in 6 points for 16 teeth for each patient, 8 maxilary teeth and 8 mandibulary, assessed with a calibrated periodontal probe marked in millimeter intervals. For the radiographic assessment we used two imaging systems: X-Ray Soredex (Minray, Soredex Palodex Group Finland) and for the CBCT scans i-CAT scanner (Imaging Science International Inc. Hatfield, PA, $120 \mathrm{kVp}, 3-8 \mathrm{~mA}, 0,2 \mathrm{~mm}$ voxel resolution $6 \times 16 \mathrm{~cm}$ field of view, 26,9 s acquisition time). All patients were informed about the risks and benefits of this investigation and they consented to participate in this study, which was approved by The Ethical Committee of Scientific Research from the University of Medicine and Pharmacy Targu Mures. The alveolar bone loss was measured in millimeters on all radiological investigations, from $1.5 \mathrm{~mm}$ below the cemento-enamel junction in 6 points on the CBCT, 3 vestibular and 3 oral. In cased with teeth restored with crowns or bridges the alveolar bone loss was measured from the gingival margin of the prosthetic components. On the conventional X-rays we selected the highest points of the alveolar bone that can be seen on the image representig the level of the alveolar bone. On CBCT images we used the millimetric ruler offerd by the CBCT software which also provides multiple angled view, 3D rotation and millimetric sections on all 3D axes.

\section{Results}

For the same patients, the images obtained on the conventional X-ray were extreamly different compared to the CBCT scans in all cases. CBCT offered the resource of exact measurement of the alveolar bone loss at least in 6 points, 3 vestibular and 3 palatal. The retroalveolar X-ray offers just a hint of the real position of the alveolar bone, exact measures of distance betwen the cemento-enamel junction or crown gingival margin and bone level could not be calculated. In all cases the alveolar bone showed by the intraoral X-ray was the oral alveolar bone, the vestibular alveolar bone being masked by the dental structures.

For the case selected, a 47 old female patient the intraoral X-ray showed the level of the alveolar bone, but not the exact periodontal situation (fig.1). All bone details were offered by the CBCT scans (fig.2,fig.3), and also the posibility of 3D rotation for measial, distal (fig.4), vestibular and oral views. The first upper premolar (2.4) exact measurement of the vestibular alveolar bone loss in 3 points were: $11.37 \mathrm{~mm}$ middle-vestibular, distal-vestibular $6.58 \mathrm{~mm}$, mezial-vestibular $7.58 \mathrm{~mm}$ (fig.2), and the oral alveolar bone measurement were: distal-palatal $6.96 \mathrm{~mm}$, mesio-palatal $6.29 \mathrm{~mm}$ and middle-palatal $6.49 \mathrm{~mm}$ as shown in fig. 3 . The same technique was used for the second upper molar (2.7). 


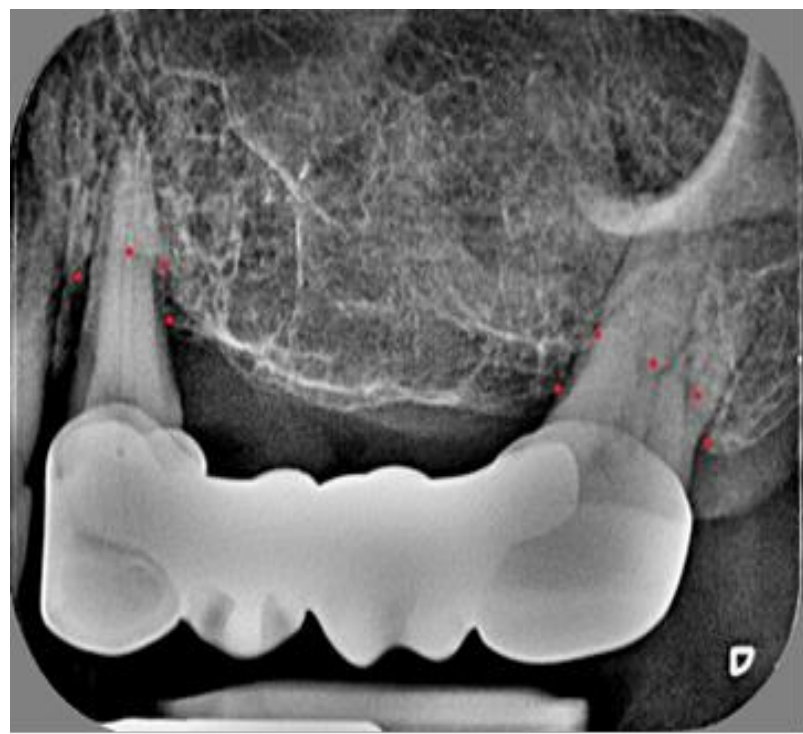

Fig.1 Digital retroalveolar X-ray - first upper premolar and second upper molar $(2.4,2.7)$, the possible position of the alveolar bone shown with red dots.

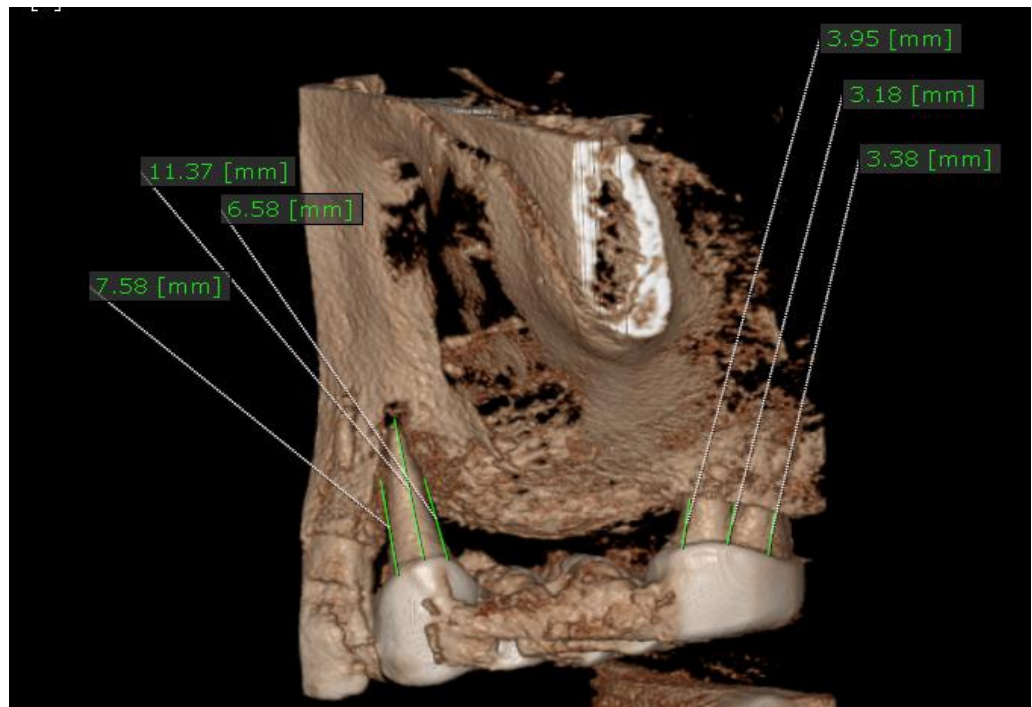

Fig.2 CBCT image - first upper premolar and second upper molar $(2.4,2.7)$, measures of the vestibular alveolar bone loss. 


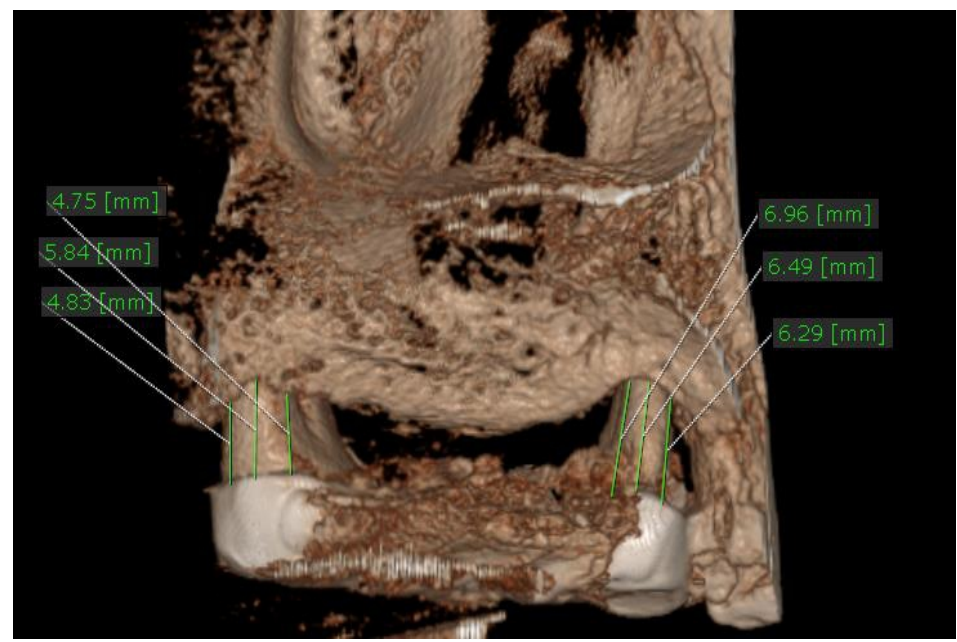

Fig.3 CBCT image - first upper premolar and second upper molar $(2.4,2.7)$, measures of the oral alveolar bone loss.

The second upper molar (2.7) exact measures of the vestibular alveolar bone loss in 3 points were: $3.18 \mathrm{~mm}$ middle-vestibular, distalvestibular $3.38 \mathrm{~mm}$, mezial-vestibular $3.95 \mathrm{~mm}$ (fig.2), and the oral alveolar bone measurement were: distal-palatal $4.83 \mathrm{~mm}$, mesio-palatal $4.75 \mathrm{~mm}$ and middle-palatal $5.84 \mathrm{~mm}$ as shown in fig.3. The 3D rotation of the CBCT software features showed the distal alveaolar bone for the second upper molar (2.7). the distance form the crown gingival margin to the alveolar bone was measured in 4 points (fig.4) from the vestibular to oral side in this order: $2.86 \mathrm{~mm}, 3.70 \mathrm{~mm}, 3.98$ and $3.30 \mathrm{~mm}$. Influencing the final diagnosis the intraoral X-ray concealed the vestibular vertical resorbtion for the first upper premolar and the furcation involvment for the second upper molar.

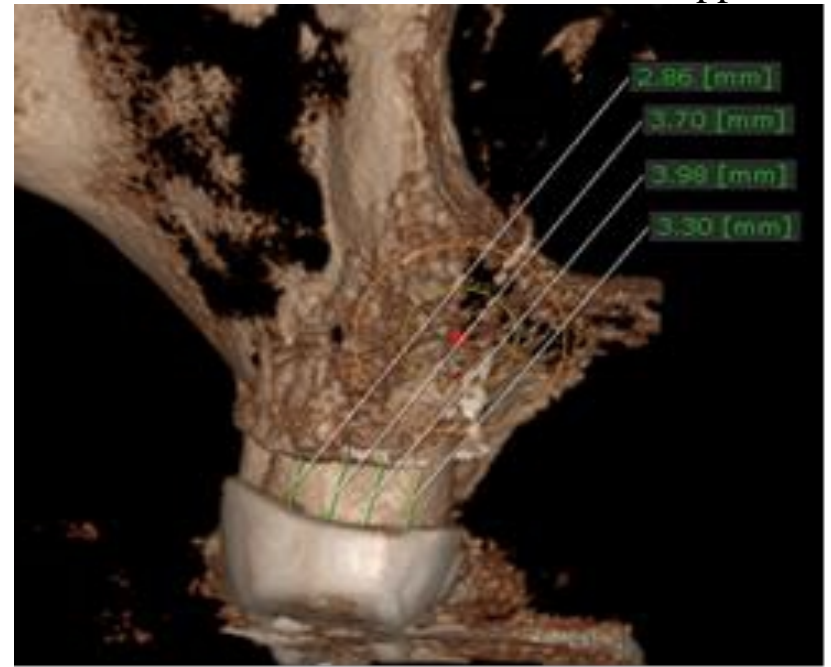

Fig.4 CBCT image - second upper molar (2.7), measurement of the alveaolar bone loss on the distal molar side. 


\section{Discussions}

The present study compared the ability of CBCT and conventional Xrays to establish the position of the alveaolar bone in Periodontitis. A few studies showed that several older tehniques that were used in the past in dental imaging as CT proved to have more disavantages (Matherne RP et al 2008, Weber MT et al 2015). CT should no longer be used for dental evaluation. Three-dimentional imaging given by CBCT delivers detailed information on the morphology of the alveaolar bone. Moreover the CBCT scans are obtained expremly fast, easy and with a lower dose of radiation compared to the CT. However, the main disavantage is the abridgement of image quality related to noise and contrast resolution because of the detection of a large quantity of dissipated radiation (Weissman $\mathrm{J}$ et al 2015). Clinicians requesting a dental CBCT should by more aware that persistent use of CBCT contributes at the increase of the total dose of radiation to the patient. A higher number of CBCT are performed on children and young adults, the matter being the higher sensitivity at radiation dose in their cases (Engström H et al 1988, Maillet M et al 2011).

The accuracy of bone measurments are essential in establishing the correct periodontal diagnosis and treatment plan, in most cases CBCT bone morphology details, changed the clinicians manner of evaluating and planning a surgical intervention. Molar teeth can be especially challenging because of the furcation involvment (Kretzschmar DP et al 2003, Shao MY et al 2009). The resorbtion of the interradicular bone is extremely difficult to evaluate on the conventional radiography almost impossible, in molar cases being considered misguiding. Radiographs could not always detect the bone defect, the measures could be unreliable beacause of the superimosition of the anatomical structures, in these cases a CBCT is required (Mafee MF et al 2006). CBCT scans can display both mesiodistal and buccolingual shape of roots and alveolar bone. Images from CBCT are anatomically correct and real proving also to be an accurate tool for linear measures of bone density shape and height. It is also helpful in follow up controls after surgical periodontal intervention (Jung YH et al 2012).

There are also other limitations besides the higher radiation dose, regarding the use of CBCT like lower image resolution, the lack of availability, cost and the presence of metal artifacts which are caused by metal and amalgam restorations, root canal fillings material and implants. Despite all these disavantages CBCT is the most usefull 3D imaging system in the present time that shows with precision all the periodontal defects including hemiseptum, infrabony defects and furcation involvment which can not be evaluated on the conventional intraoral radiography (Falk $\mathrm{H}$ et al 1986). 


\section{Conclusion}

CBCT proved extremely efficient in periodontal therapy regarding the identification of anatomic position of the alveolar bone and the follow-up controls. It was more accurate compared to conventional radiography and the differences were more evident in all cases.

CBCT must be carried out only after the medical history and clinical examination are completed.

A correct periodontal diagnosis using conventional radiography is not possible beacause of the superimposition of the anatomical structures. The importance of CBCT imaging is no longer disputed, at the present time it is the best radiographic investigation available.

\section{References:}

Patel S. New dimensions in Endodontic imaging. Part 2. Cone-beam computed tomography. Int Endod J, 2009, 42 (6): 463-475.

Durack C, Patel S. Cone beam computed tomography in endodontics. Braz Dent J, 2012; 23 (3): 179-191.

Tyndall DA, Rathore S. Cone -Beam CT Diagnostic Applications: Caries, Periodontal Bone Assessment and Endodontic Applications. Dent Clin N Am. 2008, 52: 825-841.

Agrawal P, Sanikop S, Patil S. New developments in tools for periodontal diagnosis. Int Dent J. 2012, 62 (2): 57-64.

Shekhar V, Shashikala K. Cone beam computed tomography evaluation of the diagnosis, treatment planning and long-term follow-up of large periapical lesions treated by endodontic surgery. Two case reports. Case Report in Dentistry, 2013, Article ID 564392, doi.org/10.1155/ 2013/ 564392.

Acar B, Kamburoglu K. Use of cone-beam computed tomography in periodontology. World J Radiol. 2014, 28; 6(5): 139-147.

Eshraghi T, McAllister N, McAllister B. Clinical applications of digital 2-D and 3-D radiography for the periodontist. J Evid Based Dent Pract. 2012, 12: 36-45.

deFaria Vasconcelos K, Evangelista KM, Rodrigues CD, Estrela C, deSousa TO, Silva MA: Detection of periodontal bone loss using cone-beam CT and intraoral radiography. Dentomaxillofac Radiol. 2012; 41: 64-69.

Matherne RP, Angelopoulos C, Kulilid JK, Tira D. Use of cone-beam computed tomography to identify root canal systems in vitro. J Endod, 2008: 34: 87-89.

Weber MT, Stratz M, Fleiner J, Schulze D, Hannig C. Possibilities and limits of imaging endodontic structures with CBCT. Swiss Dental Journal , 2015, SSO 125(3): 293-302.

Weissman J, Johnson JD, Anderson M, Hollender L, Huston T, Paranipe A, Patel S, Cohenca N. Association between the presence of Apical 
Periodontitis and clinical symptoms in Endodontic Patients using Cone-beam Computed Tomography and Periapical Radiographs. J Endod. 2015, Nov. 41 (11): 1824-9. doi: 10.1016/j.joen. 2015. 06. 004, Epub. 2015 sep5.

Engström H, Chamberlain D, Kiger R, Egelberg J. Radiographic evaluation of the effect of initial periodontal therapy on thickness of the maxillary sinus mucosa. J Periodontol. 1988;59:60.

Maillet M, Bowles WR, Mc Clanahan SL, John MT, Ahmad M. Cone-beam computed tomography evaluation of maxillary sinusitis. J Endod. 2011;37:753-7.

Kretzschmar DP, Kretzschmar JL. Rhinosinusitis: review from a dental perspective. Oral Surg Oral Med Oral Pathol Oral Radiol Endod. 2003;96:128-35.

Shao MY, Huang P, Cheng R, Hu T. Interleukin-6 polymorphisms modify the risk of periodontitis: A systematic review and meta-analysis. J Zhejiang UnivSci B. 2009;10:920-7.

Mafee MF, Tran BH, Chapa AR. Imaging of rhinosinusitis and its complications: plain film, CT, and MRI. Clin Rev Allergy Immunol. 2006;30:165-86.

Jung YH, Cho BH. Assessment of the relationship between the maxillary molars and adjacent structures using cone beam computed tomography. Imaging Sci Dent. 2012;42:219-24.

Falk H, Ericson S, Hugoson A. The effects of periodontal treatment on mucous membrane thickening in the maxillary sinus. J Clin Periodontol. 1986;13:217-22. 